\title{
Complex systems: \\ the amazing cross-disciplinary journey of statistical physics
}

DOI: https://doi.org/10.1051/epn/2022103

J he 2021 Nobel prize in Physics honours three outstanding scientist who, broadly speaking, have been working on complex systems: The decision of the Nobel prize committee was setting a signpost for the importance of modelling, understanding and tackling climate change (the work of Manabe and Hasselmann), and for the theoretical modelling and understanding of complex systems in general (the work of Parisi). See Europhysics News 52/5 for more detailed information on the prize winners and their work.

The current EPN issue contains two articles of $\mathrm{K}$. Binder and M. Mezard related to spin glasses - one of the major cornerstones of Parisi's work in the 1980s. These systems can be regarded as physical examples of complex systems that have played an important role in the historical development of statistical physics, exhibiting typical features such as power-law decay of correlations and very long relaxation times. Glasses are in a kind of permanent nonequilibrium state. So are many complex systems, driven by external forces and influences in a heterogeneous way, which are these days investigated in a variety of sub-disciplines.

Methods used by the Nobel prize winners are highly cross-disciplinary and universally applicable and often based on stochastic modelling via stochastic differential equations. For example, the famous Parisi-Wu stochastic quantization method is just reducing path integrals (of utmost relevance in any quantum field theory) to expectation values over higher-dimensional Brownian motion trajectories in a fictitious time coordinate-thus connecting Fokker-Planck and Langevin equations used in classical nonequilibrium problems to quantum field theory. Similarly, the work of Hasselmann uses stochastic differential equations to model climate change, where the short-scalefluctuations (modelled by noise in the stochastic differential equation) corresponds to short-scale weather effects influencing the long-term climate dynamics.

The decision of the Nobel prize committee for the 2021 Physics prize, in a sense, signals what statistical physics, in its generalized sense, has evolved to in recent decades:Towards a highly cross-disciplinary science with applications not only in physics, but connecting many different areas of science, relevant for the most important topics such as climate change that need to be solved to guarantee a sustainable future.

Environmental issues such as climate tipping points, air pollution dynamics, the dynamics of sustainable power grids, or the infection dynamics of the Covid-19 pandemic, draw in crowds of the next generations of statistical physicists, for good reasons, as this research is of utmost interest to guarantee a healthy and sustainable environment for the future of mankind, and at the same time produces highly interesting theoretical research aspects. Statistical physics methods are also used to understand cities as complex systems, as well as the dynamics of living organisms (see Europhysics News 51/5) and one could continue this list of outstanding new applications. One thing is clear: The days where statistical physics was just used to describe molecules in a gas are over. Now the relevant constituents are agents, people, renewable energy sources, traffic patterns, vehicle flows, complex biomolecules, and the interactions at macroscopic level are social contacts, communications, infections, and so on. Statistical analysis of data-driven research, complex network topologies, neural networks and modern machine learning algorithms provide a powerful universal language, helping to optimize the real-world systems under consideration.

The conferences and prizes of the EPS Statistical and Nonlinear Physics Division reflect these changes. The 2021 EPS Statistical and Nonlinear Physics prize, awarded in September 2021 during the EPS conference "Statistical Physics of Complex Systems" at ICTP/SISSA in Trieste went to A.-L. Barabási (who has pioneered Complex Network Science and its cross-disciplinary applications) and A. Vulpiani (who has pioneered Nonlinear Physics, some of his work actually jointly with Parisi). For sure the journey towards outstanding cross-disciplinary applications will continue with new and unexpected scientific discoveries by the next generation of statistical physicists.

- Christian Beck, Chair of the Statistical and Nonlinear Physics Division 